Revue internationale P.M.E.

Économie et gestion de la petite et moyenne entreprise

Revure

internationale

PME

\title{
Caractéristiques et dynamique de l'équipe dirigeante dans une jeune entreprise en hypercroissance
}

\section{Martine Séville et Peter Wirtz}

Volume 23, numéro 3-4, 2010

URI : https://id.erudit.org/iderudit/1012493ar

DOI : https://doi.org/10.7202/1012493ar

Aller au sommaire du numéro

Éditeur(s)

Presses de l’Université du Québec

ISSN

0776-5436 (imprimé)

1918-9699 (numérique)

Découvrir la revue

Citer cet article

Séville, M. \& Wirtz, P. (2010). Caractéristiques et dynamique de l'équipe

dirigeante dans une jeune entreprise en hypercroissance. Revue internationale P.M.E., 23(3-4), 43-70. https://doi.org/10.7202/1012493ar

\section{Résumé de l'article}

Cet article s'attache à comprendre le lien entre la dynamique de l'équipe de direction, ses caractéristiques et le processus d'hypercroissance. Un cadre théorique fondé à la fois sur la théorie des échelons supérieurs - de première (Hambrick et Mason, 1984) et de seconde génération (Smith et al. 1994 ; Hambrick, 2007) - et sur la théorie de la croissance de la firme de Penrose (1959) nous amène à inverser le sens traditionnellement admis de la relation entre équipe dirigeante et hypercroissance, et à considérer la dynamique de l'équipe de direction comme le résultat d'un apprentissage collectif émergeant de l'hypercroissance. L'analyse approfondie du cas d'une jeune entreprise à très forte croissance fournit une illustration concrète des propositions théoriques et révèle un apprentissage dont le coût psychologique est élevé devant l'ampleur de la tâche, lorsque la croissance est particulièrement rapide. Elle suggère, par ailleurs, l'existence d'un schéma séquentiel de diversification des expériences fonctionnelles et de la professionnalisation des services managériaux, provoquant une augmentation progressive de l'hétérogénéité au sein de l'équipe et une réallocation des tâches parmi ses membres.
Ce document est protégé par la loi sur le droit d'auteur. L’utilisation des services d’Érudit (y compris la reproduction) est assujettie à sa politique d'utilisation que vous pouvez consulter en ligne.

https://apropos.erudit.org/fr/usagers/politique-dutilisation/ 


\title{
Caractéristiques et dynamique de l'équipe dirigeante dans une jeune entreprise en hypercroissance ${ }^{1}$
}

Martine SÉVILLE

Peter WIRTZ ${ }^{2}$

Université Lumière Lyon II, France

MOTS CLÉS

Équipe de direction-Apprentissage collectif-

Hypercroissance-Théorie des échelons supérieurs-Penrose

\begin{abstract}
LES AUTEURS
MARTINE SÉVILLE est professeure des universités en sciences de gestion à I'Université Lumière Lyon II, et membre du laboratoire Coactis. Ses travaux de recherche portent sur la stratégie des entreprises, avec un intérêt particulier pour le management des connaissances, l'apprentissage organisationnel et l'action des dirigeants. Adresse: Université Lumière Lyon II, 16, quai Claude- Bernard, 69007 Lyon. Courriel : < Martine.Seville@univ-lyon2.fr>.

PETER WIRTZ est professeur des universités en sciences de gestion à l'Université Lumière Lyon II, et membre du laboratoire Coactis. Ses travaux de recherche se focalisent sur la gouvernance d'entreprise en relation avec les questions de financement et de création de valeur, avec un accent particulier sur l'approche dite «cognitive» de la gouvernance. Adresse: Université Lumière Lyon II, 16, quai Claude-Bernard, 69007 Lyon. Courriel :<peter.wirtz.fcs@gmail.com>.
\end{abstract}

\section{RÉSUMÉ}

Cet article s'attache à comprendre le lien entre la dynamique de l'équipe de direction, ses caractéristiques et le processus d'hypercroissance. Un cadre théorique fondé à la fois sur la théorie des échelons supérieurs-de première (Hambrick

1. Cette recherche a été réalisée dans le cadre du projet ANR HYPERCROIS ( $\mathrm{n}^{\circ}$ ANR-07-ENTR-008) sur «Les entreprises moyennes en hypercroissance: trajectoires et temporalités».

2. Les auteurs remercient les participants du séminaire COACTIS pour leurs remarques et suggestions. Les deux évaluateurs anonymes, ainsi que les rédacteurs du numéro spécial, Olivier Germain, Didier Chabaud et Alain Fayolle, ont contribué à une amélioration significative du texte. Qu'ils en soient remerciés. 
et Mason, 1984) et de seconde génération (Smith et al. 1994; Hambrick, 2007)et sur la théorie de la croissance de la firme de Penrose (1959) nous amène à inverser le sens traditionnellement admis de la relation entre équipe dirigeante et hypercroissance, et à considérer la dynamique de l'équipe de direction comme le résultat d'un apprentissage collectif émergeant de l'hypercroissance. L'analyse approfondie du cas d'une jeune entreprise à très forte croissance fournit une illustration concrète des propositions théoriques et révèle un apprentissage dont le coût psychologique est élevé devant l'ampleur de la tâche, lorsque la croissance est particulièrement rapide. Elle suggère, par ailleurs, l'existence d'un schéma séquentiel de diversification des expériences fonctionnelles et de la professionnalisation des services managériaux, provoquant une augmentation progressive de l'hétérogénéité au sein de l'équipe et une réallocation des tâches parmi ses membres.

\begin{abstract}
This article investigates the link between top management team (TMT) features and dynamics and the process of hypergrowth. The combined approaches of upper echelons theory and Penrose's theory of the growth of the firm give rise to a theoretical framework which predicts an inversion of the relationship between TMT and hypergrowth as a result of collective learning. The analysis of the case of a young high-growth firm yields an illustration of the conceptual frame and reveals that learning takes place at a high psychological cost when the firm grows at a particularly rapid pace. The case results also suggest that the diversification of functional experience inside the TMT follows a sequential pattern.
\end{abstract}

\title{
RESUMEN
}

Este artículo trata de explicar la relación existente entre la dinámica del equipo directivo, sus características y el proceso de hipercrecimiento empresarial. Un marco teórico basado tanto en la teoría de los escalafones superiores-de primera (Hambrick y Mason, 1984) y de segunda generación (Smith et al. 1994 ; Hambrick, 2007) - y la teoría del crecimiento de la empresa de Penrose (1959) nos lleva a invertir la relación tradicionalmente aceptada entre el equipo de gestión y el hipercrecimiento empresarial, y a considerar la dinámica del equipo de gestión como el resultado de un aprendizaje colectivo producto del hipercrecimiento. Un análisis detallado del caso de una joven empresa con muy fuerte crecimiento ilustra de manera concreta las propuestas teóricas y demuestra una enseñanza de alto costo psicológico, dada la magnitud de la tarea, cuando el crecimiento se da de manera particularmente acelerada. También se aborda la existencia de un patrón secuencial de diversificación de las experiencias funcionales y de la profesionalización de los servicios de gestión, lo que provoca un aumento gradual de la heterogeneidad dentro del equipo y una reasignación de tareas entre sus miembros. 


\section{ZUSAMMENFASSUNG}

Dieser Artikel untersucht den Zusammenhang zwischen der Dynamik der Geschäftsführung, deren Merkmalen und dem Prozess des Überwachstums. Der theoretische Rahmen, der sowohl auf der Upper Echelons-Theorie der-der ersten (Hambrick und Mason, 1984) und der zweiten Generation (Smith et al. 1994; Hambrick, 2007)-und dem Penrose -Theorem des Unternehmenswachstums (1959) fußt, veranlasst uns, die bisher anerkannte Beziehung zwischen Geschäftsführung und Überwachstum umzukehren und die Dynamik der Geschäftsführung als das Ergebnis eines durch das Überwachstum bedingten kollektiven Lernprozesses zu betrachten. Eine tiefgreifende Untersuchung des Falls eines jungen Unternehmens mit starkem Wachstum veranschaulicht konkret die theoretischen Postulate und lässt einen Lernprozess erkennen, der angesichts des Umfangs der Aufgabe während des rapiden Firmenwachstums psychologisch einen hohen Preis fordert. Die Ergebnisse dieser Fallstudie lassen auch erkennen, dass die Diversifizierung der funktionellen Erfahrungen und die Professionalisierung der Managementleistungen innerhalb der Geschäftsführung nach einem sequentiellen Muster verlaufen, was zu einer zunehmenden Heterogenität innerhalb des Führungsteams führt und eine Umverteilung der Aufgaben zwischen dessen Mitgliedern zur Folge hat.

\section{Introduction}

Les jeunes firmes en croissance d'aujourd'hui sont les grandes entreprises de demain. Elles contribuent ainsi à l'augmentation et au renouvellement du tissu économique et constituent un enjeu de premier plan pour la politique économique (Betbèze et Saint-Étienne, 2006). Pour autant, à ce jour, la dynamique de la croissance reste insuffisamment comprise. Il existe, en effet, des différences notables et non encore expliquées dans la vitesse à laquelle différentes entreprises parcourent leur cycle de croissance et, plus particulièrement, dans la phase qui peut être assimilée à leur «adolescence». Cette dernière conduit parfois à un rythme effréné, source de pressions et d'épuisement pour les équipes, dont les compétences spécifiques peuvent se trouver en inadéquation avec les exigences de plus en plus grandes imposées par la croissance. Or, l'observation montre que certaines entreprises assument mieux ce phénomène que d'autres (Hambrick et Crozier, 1985). Cela nous amène à nous interroger, certes, sur les éléments qui déclenchent l'hypercroissance, mais aussi sur les effets de cette dernière une fois qu'elle a été initiée.

Dans son ouvrage classique sur la «théorie de la croissance de la firme», Penrose (1959) consacre des développements étendus aux caractéristiques psychologiques des membres, ainsi qu'à la dynamique des interactions au sein de l'équipe dirigeante. Pour elle, c'est l'étendue des compétences entrepreneuriales et managériales de l'équipe dirigeante qui circonscrit le potentiel de croissance. La composition (Weinzimmer, 1997) et les interactions 
(Penrose, 1959; Bourgeois et Eisenhardt, 1988) au sein de l'équipe dirigeante apparaissent donc comme des clés fondamentales à la compréhension de l'hypercroissance.

Pour étudier l'influence de l'équipe dirigeante sur la trajectoire stratégique d'une firme et sur sa performance, Hambrick et Mason (1984), puis Hambrick (2007) ont développé la théorie des échelons supérieurs (UET pour upper echelons theory). Dans ce cadre, les résultats de l'entreprise, et notamment sa croissance (Weinzimmer, 1997), seraient le reflet des caractéristiques, démographiques et cognitives, de ses principaux dirigeants. L'inconvénient de l'UET est cependant son caractère relativement statique. La dynamique de l'interaction des membres de l'équipe au sein du processus stratégique, ainsi que l'impact potentiel de cette interaction sur l'acquisition et le développement des ressources cognitives au cours du temps (Penrose, 1959) y sont relativement peu abordés.

Nous proposons donc, tout en nous appuyant sur le référentiel de l'UET, de développer un cadre conceptuel qui tient compte de cette dynamique de l'équipe et de son impact sur l'évolution de ses ressources cognitives et organisationnelles dans le temps. Nous montrons que, tout en jouant un rôle certain dans le déclenchement du processus d'hypercroissance, l'équipe dirigeante est, à son tour, fortement influencée par ce processus. Il y a donc inversion du sens de la relation entre les caractéristiques de l'équipe dirigeante et la croissance. Plus cette dernière est rapide, plus l'exigence liée à l'accomplissement de la tâche managériale qui pèse sur l'équipe de direction est élevée. Il en résulte théoriquement un processus d'apprentissage difficile, marqué par des erreurs, dues à un manque de recul suffisant pour consolider les très nombreuses expériences acquises dans un laps de temps très court. La contribution centrale du présent article consiste donc à mettre en lumière l'inversion de la relation entre TMT et croissance, et de permettre de mieux comprendre les causes d'un coût psychologique élevé (sous forme d'un apprentissage difficile) dans le cas particulier de l'hypercroissance.

Les enjeux d'une telle approche de l'équipe dirigeante, étendue à sa dimension dynamique et résumée sous forme de propositions heuristiques, sont illustrés par une étude de cas.

Bien entendu, d'autres acteurs (investisseurs, administrateurs...), ressources (financières, organisationnelles...) ou mécanismes que la seule équipe dirigeante sont susceptibles d'influencer et de permettre d'assumer un rythme de croissance soutenu. Tel est notamment le cas des apports financiers et en compétences de certains acteurs du capital risque (Engel, 2002; Engel et Keilbach, 2007; Glachant, Lorenz et Trainar, 2008; Sapienza et Manigart, 1996), qui permettent le cas échéant de combler certains déficits au niveau 
des ressources détenues par les fondateurs. Ce peut être également certains mécanismes de gouvernance, tel le conseil d'administration, qui peuvent jouer le rôle de levier cognitif de l'hypercroissance (Wirtz, 2009). Les vecteurs de la forte croissance sont donc potentiellement nombreux, et leurs effets sont susceptibles d'entretenir des relations complexes: opposition pour certains, substituabilité, voire complémentarité pour d'autres. Compte tenu de la difficulté à traiter ce système complexe, le présent article se focalise exclusivement sur l'équipe dirigeante.

Dans la première partie, nous proposons une synthèse succincte et critique de l'UET, en suggérant de la compléter par la théorie de la croissance de Penrose (1959), car le dépassement d'une optique purement démographique du top management team (TMT) semble indispensable pour mieux comprendre le fonctionnement de ce dernier tout au long du processus de croissance, de même que l'examen de la manière dont la croissance peut affecter, à son tour, les ressources du TMT. Deux équipes dirigeantes peuvent, en effet, selon Penrose, avoir hérité des mêmes ressources cognitives, sans que ces ressources soient utilisées pour rendre les mêmes services productifs (productive services). Or, c'est précisément leur utilisation spécifique qui détermine, selon Penrose, la façon dont les ressources du TMT vont influencer la croissance et vice versa. Cela nous permet d'amorcer une réflexion au sujet de la nature de l'apprentissage fait par le TMT engagé dans une dynamique d'hypercroissance. Dans la deuxième partie, cette réflexion est illustrée par une étude de cas approfondie, qui concerne une entreprise ayant connu une période d'hypercroissance soutenue pendant 12 ans, d'un secteur d'activité à faible intensité capitalistique, et cela, pour exclure l'influence potentielle d'autres acteurs que les membres du TMT (tels que d'éventuels capitalrisqueurs ou des administrateurs indépendants) susceptibles d'interagir avec la croissance.

\section{1. Équipe dirigeante et hypercroissance: vers un cadre conceptuel}

\subsection{L'UET et la croissance des firmes}

Selon les principes de la théorie des échelons supérieurs (UET), ce sont les caractéristiques de l'équipe de direction qui définissent les visions et hypothèses formulées par cette équipe, elles-mêmes à l'origine de choix stratégiques et donc de la performance de l'entreprise (Hambrick et Mason, 1984; Hambrick, 2007). 
Dans les modèles inspirés de l'UET de première génération (tels que les qualifient Carpenter, Geletkanycz et Sanders, 2004), les caractéristiques évoquées sont avant tout démographiques, certaines d'entre elles ayant un lien explicite avec la croissance de l'entreprise (Hambrick et Mason, 1984). La jeunesse de l'équipe managériale serait ainsi positivement corrélée avec la croissance globale de l'entreprise (Hambrick et Mason, 1984, p. 198). A contrario, une équipe de direction composée de directeurs âgés (Hambrick et Mason, 1984; Finkelstein et Hambrick, 1990; Hambrick, Geletkanycz et Fredrickson, 1993) serait moins en mesure de saisir les idées nouvelles, donc plus favorable au statu quo et à des stratégies moins risquées. De même, l'accumulation d'expérience fonctionnelle de l'équipe dans des fonctions externes (output functions), telles que le marketing, la vente et la recherche et développement, favoriserait davantage la croissance de l'entreprise qu'une expérience fonctionnelle dans des fonctions centrées sur le fonctionnement interne, telles que la production et la comptabilité. Plus généralement, c'est l'hétérogénéité de l'équipe qui pourrait être une source de croissance comme l'ont souligné Eisenhardt, Schoonhoven et Bird (1990) puis Hambrick, Cho et Chen (1996). L'hétérogénéité de l'équipe accroît le nombre d'alternatives qu'elle considère et enrichit leur processus d'évaluation (Hambrick, Cho et Chen, 1996; Boeker, 1997). Elle est ainsi une source d'enrichissement et de variété des actions entreprises. Elle ralentit toutefois la production d'une réponse collective par cette équipe et son exécution (Hambrick et al.,1996). L'hétérogénéité serait donc une épée à double tranchant: d'une part, elle élargirait les perspectives de l'équipe de direction et, d'autre part, elle serait une source de dissension et d'inefficience potentielle.

Les caractéristiques démographiques ne permettent pas toutefois d'épuiser la dimension cognitive des équipes de direction (Smith et al., 1994; Kilduff, Anglemar et Meehra, 2000; Carpenter et al., 2004). Deux membres d'une équipe aux expériences fonctionnelles identiques peuvent, en effet, différer dans leurs visions cognitives, en fonction de leurs buts et de leurs valeurs (Olson, Parayitam et Bao, 2007) ou selon les mécanismes à l'œuvre dans les équipes. Un certain nombre d'auteurs (Kilduff et al., 2000; Carpenter et al., 2004; Peterson et al., 2003; Olson et al., 2007) vont, dès lors, dans des modèles de seconde génération, plus dynamiques et cognitifs, aller au-delà de la seule démographie de l'équipe, pour s'intéresser aux processus de fonctionnement de l'équipe et à des variables psychologiques, telles que la satisfaction des membres, le degré d'adhésion aux valeurs, la distribution du pouvoir politique intra-équipe (Kilduff et al., 2000; Carpenter et al., 2004; Greve et Mitsuhashi, 2008) et la confiance intragroupe (Olson et al., 2007), ou encore aux critères de sélection et aux systèmes d'incitations (Lin et Shih, 2008). Au-delà des seules caractéristiques démographiques, c'est 
donc l'ensemble des variables qui peuvent influencer la rapidité et la qualité des décisions prises par les équipes de direction qui vont attirer l'attention des chercheurs (Lin et Shih, 2008). On retrouve, en ce sens, dans ces travaux un point commun: la mise en évidence pour les organisations qui veulent changer, se développer et croître, d'exigences contradictoires de consensus et de conflits, d'hétérogénéité et d'homogénéité cognitive (Eisenhardt et al., 1990; Kilduff et al., 2000; Olson et al.,2007). L'équipe du top management doit, en effet, utiliser la diversité cognitive pour augmenter la qualité des décisions (Amason, 1996), tout en préservant le consensus de base, la confiance et l'acceptation des décisions parmi ses membres. C'est le paradoxe de l'équipe managériale (Amason, 1996); paradoxe que l'on pourrait retrouver très prégnant dans le cas d'une équipe de direction d'une jeune entreprise en hypercroissance.

\section{2. Équipe de direction et hypercroissance: proposition d'un cadre dynamique}

L'hypercroissance est une forme intensive et très rapide de croissance, dans laquelle les décideurs font face à une situation particulièrement complexe et qui évolue à grande vitesse. L'équipe de direction doit prendre des décisions dans un laps de temps limité en évaluant les différentes alternatives qui s'offrent à elle, tout en gérant les nouvelles dimensions organisationnelles et stratégiques d'une entreprise, dont la taille croît instantanément et qui se métamorphose (Hambrick et Crozier, 1985). Cette métamorphose correspond à un processus de changement, et la reconnaissance de ce caractère processuel du phénomène d'hypercroissance, sous-estimé par les modèles de l'UET, y compris de seconde génération qui restent globalement statiques, nous apparaît comme un enjeu fondamental pour sa bonne compréhension. On notera toutefois que, si la théorie de l'UET s'intéresse avant tout à l'influence des antécédents managériaux sur les résultats de la firme, elle reconnaît également que ces antécédents managériaux peuvent eux-mêmes être influencés par des facteurs environnementaux, tels que le degré de régulation de l'industrie ou l'importance de la croissance de l'industrie (Hambrick et Mason, 1984). La théorie admet donc que les caractéristiques des équipes managériales ne sont pas dues au hasard, mais peuvent être le résultat de choix stratégiques préalables. La causalité - caractéristiques des équipes managériales - choix stratégiques et résultats - peut donc jouer dans les deux sens (Hambrick et Mason, 1984), sans remettre en cause l'UET. Hambrick et al., en 2005, puis Hambrick en 2007, suggèrent d'ailleurs de «tourner la théorie dans l'autre sens », en étudiant les caractéristiques de l'équipe de direction comme une conséquence plutôt que comme une cause des choix stratégiques, laissant ainsi ouverte, nous semble-t-il, la possibilité d'adopter une approche 
plus processuelle des phénomènes de croissance. Carpenter et al. (2004, p.28) concèdent, en effet: «Nous manquons d'une perspective évolutionniste du modèle des échelons supérieurs. Clairement, les perspectives et attributs des membres de l'équipe de direction ne sont pas statiques, mais changent au cours du temps. [...] Une telle perspective nécessite des méthodes longitudinales et qualitatives. » C'est précisément à cet appel pour une approche davantage processuelle des caractéristiques et du fonctionnement du TMT que nous souhaitons répondre. Or, appréhender l'hypercroissance comme un processus rend l'explication potentiellement plus complexe, dans la mesure où rien ne garantit que les différentes variables démographiques et cognitives jouent le même rôle aux différents stades du processus. Nous nous intéressons donc à la fois au rôle de l'expérience de l'équipe de direction dans le processus d'hypercroissance et aux conséquences de l'hypercroissance pour la dynamique du TMT, en analysant à quels stades plus précisément ces effets interviennent, à travers un jeu de quatre propositions présenté ci-dessous.

Toutes les aventures entrepreneuriales ne conduisent pas à une croissance effrénée. Ainsi, la recherche d'une forte croissance continue n'est pas un objectif privilégié par tous les entrepreneurs. Pour Kor (2003), c'est le groupe de fondateurs au sein de l'équipe dirigeante qui jouerait un rôle central pour expliquer l'importance de la croissance. Leur expérience spécifique influencerait, en effet, leur capacité à repérer et à saisir des opportunités stratégiques de croissance. On peut donc supposer que le noyau fondateur de l'équipe dirigeante joue un rôle fondamental aux premiers stades de la vie d'une firme entrepreneuriale pour initier le cycle de l'hypercroissance, grâce au repérage et/ou à la conception des opportunités du marché. L'expérience spécifique du noyau fondateur de l'équipe dirigeante jouerait ainsi un rôle déclencheur du phénomène d'hypercroissance par sa capacité à concevoir des opportunités de croissance.

Plus précisément, selon Penrose (1959, p. 32), les opportunités de croissance n'existent pas indépendamment des principaux dirigeants. Elles sont limitées par la plus ou moins grande capacité des dirigeants de les voir, ainsi que par leur volonté de les saisir. Penrose (1959) distingue les ressources entrepreneuriales et managériales «héritées» (p. 48) (c'est-à-dire existant à un moment donné, indépendamment du processus de croissance) et celles acquises par l'expérience au cours du processus de croissance. Les premières sont une condition importante pour le développement des secondes et jouent, donc, vraisemblablement un rôle fondamental dans le déclenchement d'un mouvement d'hypercroissance. Elles concernent à la fois les capacités cognitives, permettant de percevoir des opportunités de croissance, et le système de valeurs de certains dirigeants-fondateurs, naturellement favorables à des objectifs de développement ambitieux. Ainsi, pour Penrose (1959, p. 36), les 
premières qualités exogènes des dirigeants fondateurs créant les conditions d'une croissance soutenue sont l'imagination et la vision, qui permettent de dépasser un horizon à très court terme. Identifier les opportunités de croissance repose en grande partie sur la perception d'un potentiel de marché pour certains types de produits ou de services. Rappelons d'ailleurs que Hambrick et Mason (1984) supposent une influence positive d'une forte expérience dans les fonctions d'output sur la croissance, ces fonctions étant les plus proches des évolutions du marché. Ainsi, c'est en combinaison avec un certain type d'expérience professionnelle (fonctions output: R\&D, commerciale, ingénieur consultant...) que les capacités visionnaires des membres fondateurs du TMT sont susceptibles d'avoir l'impact le plus fort sur l'ampleur et la durée du phénomène de croissance.

P1: Une expérience spécifique, notamment des fonctions output, du noyau fondateur du TMT, combinée avec une vision imaginative de long terme des besoins d'une clientèle potentielle, favorise le déclenchement d'un cycle d'hypercroissance soutenu, en aidant à la conception d'opportunités de croissance.

Au-delà des ressources cognitives et financières, les valeurs prioritaires des dirigeants façonnent vraisemblablement leurs choix (Hambrick et Mason, 1984). Relever le défi d'une croissance soutenue à deux chiffres dans la durée signifie que le développement de l'affaire est considéré comme une priorité. Cela suppose une certaine dose d'ambition et l'acceptation des efforts nécessaires pour constamment changer de taille à un rythme soutenu. Penrose (1959, p. 39; traduction libre) qualifie les dirigeants-fondateurs qui agissent sur la base de telles valeurs (valorisation de l'effort pour un développement ambitieux) de «constructeurs de good-will»: «Leur intérêt est focalisé sur l'amélioration de la qualité de leurs produits, sur la réduction des coûts, le développement d'une meilleure technologie, une extension du marché grâce à un meilleur service rendu aux clients, et l'introduction de nouveaux produits [...] Ils sont fiers de leur organisation.» Il est possible de rapprocher une telle attitude de ce que Hambrick, Finkelstein et Mooney (2005, p. 477-478) qualifient d'un haut degré d'aspirations managériales ${ }^{3}$, dont on peut supposer une influence positive sur la volonté de croître.

$P 2$ : À tous les stades, l'hypercroissance est favorisée par la priorité accordée au développement et à la valorisation de l'effort au sein du système de valeurs caractérisant le noyau fondateur du TMT.

3. Notons que, selon Hambrick et al. (2005), les aspirations managériales sont l'un des déterminants du concept d'exigence de la fonction managériale. Il s'agit en quelque sorte d'une exigence auto-imposée. 
Une fois enclenché, le processus d'hypercroissance conduit à des changements rapides de taille de l'entreprise, susceptibles d'influencer la nature des relations entre ses différents membres, au-delà de certains seuils. Ainsi, l'expérience de la croissance peut être supposée, à son tour, avoir un impact sur les caractéristiques, notamment en termes de compétences requises, de l'équipe managériale. L'utilisation des ressources managériales héritées dans l'accomplissement des tâches particulières liées à la croissance conduit donc théoriquement à l'acquisition de ressources cognitives nouvelles (Penrose, 1959) qui, elles-mêmes, selon l'utilisation qui en est faite, vont entraîner la croissance. Le défi d'une inversion du sens de causalité entre résultats organisationnels (notamment la croissance) et caractéristiques du TMT a récemment été lancé par Hambrick (2007, p. 338; traduction libre): «Il est nécessaire d'inverser la théorie des échelons supérieurs en considérant les caractéristiques des dirigeants comme des conséquences plutôt que comme des causes. [...] Pourquoi l'équipe de direction se présente-t-elle comme elle le fait? Quels sont les facteurs qui conduisent au changement du profil du TMT? » Nous suggérons que les spécificités de la dynamique de croissance contribuent à expliquer le changement de l'équipe dirigeante, aussi bien dans sa composition que dans les rôles assumés par ses différents membres.

P3: L'expérience acquise dans l'avancement du processus de croissance conduit le noyau fondateur du TMT à percevoir la nécessité d'acquérir des ressources cognitives supplémentaires. Cela induit un changement de la composition, des compétences et de la répartition des rôles de l'équipe dirigeante.

Peu de travaux de l'UET étudient le management du processus de croissance en tant que tel, se focalisant sur le seul processus de conception des choix stratégiques (ex. Kor, 2003). Or, Hambrick et Crozier (1985) montrent que la dynamique d'une très forte croissance exige des aptitudes particulières de la part de l'équipe dirigeante et de sa gestion.

Pour eux, l'une des difficultés, sur le plan managérial, d'une croissance particulièrement rapide, est le changement quasi instantané de taille (p.35-36). Ils rappellent à ce sujet que les aptitudes managériales pour gérer une entreprise de 100 personnes ne sont pas les mêmes que celles pour une entreprise de 1000 personnes. Ces aptitudes peuvent être acquises par l'expérience de travail du TMT, et le processus d'expansion contribue ainsi à créer des compétences managériales nouvelles (Penrose, 1959, p. 51). Or, la construction de compétences par l'expérience de travail comporte une dimension temporelle. Penrose (1959, p. 203-204) admet qu'un pas de croissance particulièrement rapide exige des ajustements tellement rapides des façons de faire que l'équipe dirigeante manque parfois du temps nécessaire pour s'y habituer et les intégrer pleinement (voir notamment p. 204, note 1). 
L'hypercroissance rend donc vraisemblablement la tâche administrative des membres du TMT particulièrement exigeante. Pour Hambrick et al. (2005), le degré d'exigence de la fonction managériale serait négativement lié à la performance. Les managers qui doivent gérer une exigence de la fonction managériale élevée (ex. la forte pression d'adaptation liée au changement instantané de la taille) vont être obligés de faire des «raccourcis mentaux» et de revenir à ce qu'ils savaient faire dans le passé, conduisant potentiellement à des décisions inadaptées au contexte de la croissance. Les dirigeants qui, au contraire, sont soumis à des exigences plus faibles (p. ex. croissance plus lente) seront plus exhaustifs dans leur analyse et plus rationnels dans leur prise de décision. Leurs choix cadreront alors mieux avec les conditions objectives de leur situation, et ils commettront moins d'erreurs de management. Concernant les décisions managériales, l'hypercroissance crée une pression pour une adaptation rapide de la forme et du fonctionnement de l'équipe dirigeante, de façon à compenser les capacités limitées de ses membres. Une telle transformation de l'équipe dirigeante peut s'opérer de deux façons, soit par l'intégration de nouveaux membres aux compétences complémentaires (notamment en termes de profil fonctionnel), soit par la réallocation des rôles parmi les membres de l'équipe pour exploiter au mieux les capacités acquises de chacun.

P4: L'hypercroissance induit un haut degré d'exigence pour l'accomplissement des tâches administratives (task challenge) faisant ressentir un besoin de recrutement pour l'équipe managériale. Le haut degré d'exigence est source d'erreurs dans la prise de décision car, par un manque de temps pour consolider leur expérience, les membres du TMT en place opèrent des "raccourcis mentaux» dans leur prise de décision (Hambrick et al., 2005).

Nous obtenons donc ainsi deux propositions (P1 et P2) portant sur l'influence des caractéristiques du TMT sur le déclenchement ou le maintien de l'hypercroissance, puis deux autres (P3 et P4) suggérant l'effet du processus d'hypercroissance sur la dynamique de l'équipe de direction.

\section{2. Étude d'un cas d'hypercroissance dans une entreprise à faible intensité capitalistique}

Une étude de cas approfondie nous permet de mettre le cadre conceptuel précédemment ébauché à l'épreuve des faits. La description du design de l'étude sera suivie d'un récit synthétique du cas (2.1.). L'exposé des résultats de l'analyse de contenu procède ensuite d'une confrontation systématique des propositions théoriques avec les données du cas (2.2.). 


\subsection{Design de l'étude et chronologie du cas}

Nous avons sélectionné une entreprise appartenant à un secteur à faible intensité capitalistique, le conseil en ingénierie. L'entreprise Service Conseil ${ }^{4}$, bien qu'ayant régulièrement doublé d'effectif par le passé, reste, pour le moment, une PME. Elle affiche des taux de croissance à deux chiffres sur une période longue. Le recueil de données qualitatives a été effectué grâce à des entretiens semi-directifs centrés, menés avec les membres de l'équipe dirigeante ${ }^{5}$. Les quatre personnes les plus haut placées dans la structure managériale (membres du TMT) ont ainsi été interviewées: le fondateur, appelé «Jérôme», et le cofondateur, appelé «Pascal» (ces deux constituant le noyau fondateur du TMT), le directeur commercial (frère du fondateur, appelé «Arthur»), et le directeur administratif et financier (DAF), appelé «M. Marco». Les entretiens, d'une durée moyenne de deux heures, enregistrés et intégralement retranscrits, ont fait l'objet d'une analyse de contenu systématique en fonction de la grille ci-contre (cf. tableau 1). Figurent en ligne les codes correspondant aux concepts clés du cadre théorique et, en colonnes, les stades du processus de croissance. Les chiffres du tableau montrent le nombre de sources, dans lesquelles les thèmes ont été repérés lors du codage, sachant que les sources correspondent aux entretiens codés par chaque codeur, ce qui donne un total de huit sources possibles (quatre entretiens et deux codeurs). On peut supposer que la présence d'un thème dans une source traduit sa pertinence aux yeux de la personne interrogée $e^{6}$. Le tableau révèle alors une pertinence inégale des différents concepts selon le stade (déclenchement ou avancement) du processus de croissance ${ }^{7}$.

Tous les entretiens ont fait l'objet d'un double codage par les coauteurs de cet article, grâce au logiciel d'analyse de contenu N'VIVO (version 7) ${ }^{8}$. À la suite du codage, une matrice d'analyse a été créée (Miles et Huberman,

4. Pour des raisons de confidentialité, des noms fictifs ont été attribués à l'entreprise et à ses dirigeants.

5. Les entretiens ont été réalisés après le franchissement d'un seuil important de l'entreprise suivant une période d'hypercroissance soutenue pendant une douzaine d'années.

6. Cette analyse part du postulat que les schémas cognitifs des membres de l'équipe dirigeante à un instant $\mathrm{T}$ sont le résultat du processus d'apprentissage des périodes précédentes et devraient ainsi porter les traces de ce processus.

7. Il s'agit d'une première indication quant à la pertinence d'une approche processuelle des échelons supérieurs.

8. Pour davantage de précisions, la grille de codage explicitant les critères d'attribution pour les codes thématiques et des exemples de verbatim codé peuvent être obtenus sur demande auprès des auteurs. 
1994), croisant les variables de type concepts clés avec les stades et enjeux du processus de croissance. Cette analyse croisée permet un rapprochement du matériau qualitatif et des propositions du cadre conceptuel.

La société Service Conseil compte 225 salariés pour un chiffre d'affaires d'environ 17 millions d'euros (au 30 juin 2008, après environ 15 millions d'euros l'année précédente). Elle a été créée en 1995 par deux amis d'études, Julien et Pascal, qui avaient également fait une partie de leur parcours professionnel ensemble, en tant que consultants dans une société de service. L'idée de la création peut être attribuée à Julien, qui percevait l'opportunité du développement d'un marché de services pour un secteur industriel très fortement réglementé. Les services proposés portaient essentiellement sur la validation des équipements et des systèmes. Au moment de la création de Service Conseil, ce marché était inexistant en France. Il a été développé par Service Conseil qui, constitué de deux personnes initialement, affichait un chiffre d'affaires de 3 millions de francs au bout de la première année, pour atteindre 17 millions d'euros aujourd'hui, avec un taux de croissance annuelle moyenne de l'ordre de $22 \%$ au cours des six dernières années. Dès son origine, la société a connu quasiment un doublement de son effectif chaque année.

TABLEAU 1

Résumé de l'analyse de contenu des sources ( 4 entretiens codés par 2 codeurs $=8$ sources)

\begin{tabular}{llll}
\hline & Tous stades & Déclenchement & Avancement \\
\hline Changement d'équipe & 8 & 3 & 8 \\
\hline Décision de réallocation des tâches & 6 & 1 & 6 \\
\hline Perception de la nécessité de recrutement & 7 & 1 & 7 \\
\hline Recrutement effectif & 8 & 2 & 8 \\
\hline Exigence de la fonction managériale & 8 & 4 & 8 \\
\hline Imposée par la croissance & 8 & 2 & 8 \\
\hline $\begin{array}{l}\text { Valorisation de soi, développement } \\
\text { et effort }\end{array}$ & 8 & 4 & 8 \\
\hline Expérience & 8 & 4 & 8 \\
\hline Expérience de croissance & 8 & 1 & 7 \\
\hline $\begin{array}{l}\text { Expérience en fonctions externes } \\
(c f . \text { expérience en output) }\end{array}$ & 6 & 3 & 4 \\
\hline Expérience périphérique & 6 & 2 & 6 \\
\hline Vision & 6 & 4 & 4 \\
\hline
\end{tabular}


Julien peut être caractérisé comme un visionnaire, avec une forte capacité d'anticipation des besoins du marché, alors que Pascal est un technicien de haut niveau, capable d'élaborer les solutions concrètes pour la mise en œuvre des idées de Julien. Étant initialement seule sur le marché, Service Conseil a pu imposer ses standards méthodologiques, mais a dû, également, s'engager dans un effort de formation très important (essentiellement assuré par Julien) au fur et à mesure des recrutements de nouveaux consultants.

Si l'on se situe sur le plan stratégique, le modèle de développement de la société a connu trois phases distinctes. La première recouvre grosso modo la seconde moitié des années 1990. Elle se caractérise par la sensibilisation des clients à l'importance de la démarche proposée. Pendant cette période, Service Conseil était seule sur le marché, et l'obtention de nouveaux contrats était facile et engendrait une croissance rapide. La taille des contrats vendus à l'époque se situait entre 50000 et 100000 euros. L'approche de l'an 2000, avec la peur du bug de l'an 2000, a ensuite fait exploser la demande, et comme Service Conseil était dans l'incapacité de faire face à l'intégralité de la demande, certains acteurs industriels se sont tournés vers des concurrents généralistes. Depuis le début des années 2000, la société s'est donc retrouvée sur un marché concurrentiel. Julien anticipait alors le besoin de modifier l'approche du marché vis-à-vis des clients potentiels. Un travail commercial plus professionnel était ressenti comme un vecteur de développement important pour les années à venir. Au cours de la première période de développement, Pascal, en effet, avait été de plus en plus impliqué dans la gestion des relations commerciales avec les clients. Pour lui permettre de se re-concentrer sur le développement technique, sa compétence première, il a été décidé d'élargir l'équipe de direction, en embauchant Arthur, le frère de Julien, qui disposait d'une expérience commerciale à l'étranger, au sein d'un groupe d'une certaine taille.

Avec l'arrivée d'Arthur, l'approche commerciale a été modifiée de façon significative. Une équipe de commerciaux a été mise en place et structurée et l'entreprise a véritablement séparé les fonctions commerciales des fonctions «conseil». Service Conseil a de plus en plus axé sa démarche commerciale sur une politique d' «écoute client » et a commencé à se développer à l'étranger, notamment en Belgique. La taille moyenne des contrats a aussi fortement augmenté, avec de gros contrats de l'ordre de 2 millions d'euros.

L'activité de validation des systèmes a ensuite conduit Service Conseil à développer un logiciel pour faciliter le travail des consultants en automatisant certains aspects de la démarche. Cela a permis d'augmenter considérablement la productivité des équipes (de l'ordre de $40 \%$ ). Cet outil avait initialement été développé pour l'usage interne, mais ensuite, la décision d'investir dans 
le développement de ce type de logiciel a été adoptée, pour qu'il puisse être commercialisé et utilisé directement par certains clients. Cette étape a été en partie poussée par la demande des clients. Cela a alors inauguré une nouvelle étape pour le développement stratégique de Service Conseil.

Sur le plan de la dynamique organisationnelle et du management, le passage de deux seuils semble avoir marqué les dirigeants. Le premier seuil a été touché vers l'an 2000, lorsque Service Conseil a atteint un effectif d'environ 50 personnes. La direction percevait à cette époque l'inadéquation de certains outils et méthodes de gestion. Cela concernait plus particulièrement la fonction finance. Ainsi, la direction s'est aperçue que le comptable qui avait été recruté, et qui avait l'expérience d'une structure d'une trentaine de personnes, était incapable de faire évoluer les outils (notamment en termes de reporting), ce qui aurait été nécessaire pour accompagner la forte croissance.

Un deuxième seuil semble avoir été atteint en 2006, au passage de la marque des 150 salariés. Dans l'intervalle, les dirigeants déclarent avoir subi les effets négatifs d'un certain nombre «d'erreurs de casting». Certains recrutements se sont, en effet, révélés particulièrement préjudiciables, notamment lors d'une tentative de développement d'une fonction de direction des ressources humaines. Les dirigeants ont alors dû investir beaucoup d'énergie dans des litiges sociaux, expérience qu'ils ont vécue comme une épreuve particulièrement pénible 9 .

La fonction financière semble être perçue comme une ressource critique au passage du seuil des 50 personnes. En même temps, les dirigeants historiques manquaient de savoir-faire pour définir le profil adéquat et attirer les compétences requises, qui auraient pu servir de facilitateur dans la gestion de l'hypercroissance. L'incapacité à faire évoluer la fonction finance a donc été ressentie comme un frein à la croissance.

L'année 2006 a ensuite été marquée par le recrutement d'un directeur administratif et financier, M. Marco. Ce dernier avait l'expérience de la direction financière de filiales (entités de 300 à 450 personnes) de grands groupes, avec la culture des processus budgétaires. Il a apporté un certain nombre de procédures davantage formalisées, permettant notamment la restructuration du système d'information financière. Le «courant était passé» très vite entre ce DAF et les dirigeants historiques, et l'embauche s'est conclue très rapidement, en l'espace de quatre jours. En fait, le recrutement, mené par simple annonce, d'un nouveau directeur financier a enfin pu apporter un savoir-faire

9. Les erreurs de casting sur un certain nombre de fonctions internes sont alors considérées comme un frein majeur à une croissance sereine. Elles sont aussi vues comme une fatalité avec l'idée que tant que l'on ne s'est pas trompé, on ne peut pas savoir bien faire. 
essentiel, en 2006. Les dirigeants historiques reconnaissent l'apport de la professionnalisation de la fonction finance et se disent aujourd'hui parés, à ce niveau au moins, pour atteindre le cap des 500 salariés. L'apport du nouveau DAF, qui a permis de compléter la base de compétences de la direction (plutôt centrées sur le développement de solutions techniques et la gestion des relations commerciales) portant son effectif à quatre personnes, s'est révélé décisif en matière de professionnalisation des méthodes de management.

\subsection{Résultats de l'analyse du cas Service Conseil}

La présente section comporte les résultats d'une interrogation systématique du matériau empirique qualitatif par rapport à chacune des propositions théoriques. Pour apprécier une proposition, nous sommes partis de la matrice d'analyse présentée supra et avons relu le verbatim lié à chaque code dont le concept figure dans la proposition. L'appréciation de la proposition P3, par exemple, a consisté à analyser la présence du code EXPÉRIENCE DE LA CROISSANCE dans le stade d'avancement du processus d'hypercroissance ainsi que son lien avec le code CHANGEMENT D’ÉQUIPE.

\subsubsection{Caractéristiques du TMT, déclenchement et maintien de l'hypercroissance}

$P 1^{10}$ : Une expérience spécifique, notamment des fonctions output, du noyau fondateur du TMT, combinée avec une vision imaginative de long terme des besoins d'une clientèle potentielle, favorise le déclenchement d'un cycle d'hypercroissance soutenu, en aidant à la conception d'opportunités de croissance.

À l'origine de la société Service Conseil et de sa très forte croissance se trouvent deux amis d'études, Julien et Pascal, qui partagent, outre leur ancienne amitié, plusieurs années d'expérience de travail en commun dans un cabinet de consultants. Cette expérience commune leur vaut une bonne connaissance du marché ainsi que des lacunes dans l'offre de services existante:

$[\mathrm{O}] n$ s'est retrouvés dans cette société de conseil et puis cette société de conseil a évolué, elle vivotait. On a vu le vieux consulting donc ce qu'on appelait les «crocodiles», puis on a vu aussi des tas de managers se succéder, qui n'écoutaient pas les consultants, qui n'écoutaient pas les clients, etc., donc, un beau jour, on en a eu assez, on voyait tous les autres gens qui se plaignaient, nous on bossait, on faisait notre boulot mais je trouvais qu'il y avait beaucoup d'énergie qui était

10. Analyse croisée des passages de texte correspondant aux codes EXPÉRIENCE OUTPUT, VISION et DÉCLENCHEMENT. 
passée à se plaindre et à attendre que tout vienne du ciel donc on a dit: Il y en a assez! Puisqu'on a vu tout ce qu'il ne fallait pas faire, maintenant on devrait savoir quoi faire, et donc on a eu l'idée de créer cette entreprise. (cofondateur)

Les deux amis s'associent, donc, et démarrent une affaire dont ils restent à ce jour les dirigeants-fondateurs. À deux, ils constituent ce qui peut être qualifié de noyau fondateur du TMT. Le travail au cœur de la conception de services informatiques pour l'industrie leur donne une bonne connaissance des pratiques dans les secteurs des entreprises clientes, que ce soit en France ou sur des marchés étrangers. Cela leur permet alors d'anticiper certains besoins du marché domestique et de projeter, par conséquent, un potentiel de développement significatif:

[J]e suis devenu consultant comme ça. Et puis après, j'ai eu plein d'idées. Et je me suis intéressé à la pharma, dans l'entreprise où j'avais été, ils s'intéressaient à la pharma. Donc je suis rentré comme ça dans la pharma. Et puis ils avaient des idées qui étaient intéressantes, et que j'ai un peu conceptualisées. (fondateur)

C'est un marché qui existait aux États-Unis, qu'on avait rencontré précédemment dans notre carrière, et auquel on pensait qu'il y avait un développement à venir. Les entreprises de la santé étaient très peu sensibilisées à ce sujet-là. On savait que ça existait, qu'il y avait des choses à faire. (fondateur)

Cette expérience spécifique du noyau fondateur lui a permis de concevoir un marché potentiel en France qui était jusque-là inexploré.

On a senti un marché [...] Il y a une première expérience et on prend un marché qui n'existe pas, on crée le marché finalement. (cofondateur)

Cette création d'un marché nouveau débouche ensuite, pour la jeune société, très rapidement sur une croissance importante.

$[\mathrm{O}] n$ a commencé à deux, et puis très rapidement, on embauche une première personne, une deuxième, une troisième, etc. On a fini l'année avec un chiffre d'affaires de 3 millions de francs, à l'époque. Je ne sais pas combien ça fait en euros, 450 000. Oui, exact, 450000 euros. Et puis après, on a doublé à peu près les effectifs tous les ans. La grosse difficulté qu'on avait, c'est qu'il n'y avait pas de ressource sur le marché. Forcément, on était sur un marché qui «n'existait pas». Il n'y avait pas de compétence. (fondateur)

Le récit des cofondateurs traduit ainsi le rôle central de leur expérience spécifique commune dans la conception d'un marché nouveau en France, avec un potentiel de développement élevé. Ce potentiel débouche rapidement sur une croissance réelle, avec un doublement des effectifs tous les ans. 
Les deux cofondateurs ont été aidés, dans cette conception d'un marché nouveau par une importante expérience output (production de services de conseil dans une SSII pendant plusieurs années) face à une clientèle industrielle.

J'ai travaillé à peu près 5-6 ans pour une société de conseil sur des process industriels. C'était assez variable. C'est à ce moment-là que j'ai rencontré des sociétés, j'ai commencé à travailler pour le secteur de l'industrie pharmaceutique. [...] j'ai toujours été intéressé par l'industrie puisque l'industrie, il y a des produits, il y a des process, c'est compliqué, il faut rechercher, il faut développer. (cofondateur)

Par ailleurs, le fondateur, Julien, est qualifié, selon plusieurs sources convergentes, d'homme à visions et de créatif: «j'ai plutôt des visions» (fondateur).

C'est cette combinaison d'une forte expérience de conseil en face d'une clientèle industrielle (source d'une bonne connaissance des besoins) et la capacité, voire l'audace, d'imaginer la possibilité de développer un marché inexistant en France, qui sont à l'origine de la création de Service Conseil et de sa très forte croissance. La vision d'un marché à bâtir était, en effet, originale à l'époque en France et n'était pas partagée par le management du cabinet de conseil qui a permis aux cofondateurs d'acquérir l'expérience professionnelle liée aux fonctions output, ce qui montre bien que cette expérience seule n'est pas suffisante pour stimuler la croissance liée à l'approche d'un marché potentiel.

Et là, je leur ai dit [à mes anciens employeurs] que si, on y croyait vachement, etc. Et ils m'ont dit: «Écoutez, si vous voulez, dégagez.»Alors on a dit: «Pas de problème, on dégage.» Et ça s'est très bien passé. (fondateur)

Par conséquent, nous considérons la proposition P1, selon laquelle l'expérience spécifique dans les fonctions output du noyau fondateur de l'équipe dirigeante joue un rôle déclencheur du phénomène d'hypercroissance, dans la mesure où elle aide à concevoir la stratégie de marché, comme corroborée. Cela valide, pour le cas du déclenchement d'une stratégie d'hypercroissance, le postulat de base de l'UET, selon lequel les stratégies des entreprises (dans lesquelles les dirigeants jouissent d'un important espace discrétionnaire) sont le reflet des caractéristiques cognitives des membres du TMT.

Au-delà de la cognition en matière de stratégie de marché, le cas attire cependant notre attention sur une autre caractéristique importante du déclenchement du processus d'hypercroissance, qui concerne plus particulièrement la capacité à saisir réellement les opportunités de croissance perçues. Il s'agit en l'occurrence d'un problème de ressources. Dans un secteur à faible intensité capitalistique, les ressources en jeu ne sont pas d'abord financières, mais humaines. Dans la mesure, où le marché permettant l'hy- 
percroissance ne préexiste pas à l'effort d'innovation du noyau fondateur, les compétences requises pour exploiter le potentiel de marché sont rares, voire quasi inexistantes. Il faut donc être en mesure de les créer. Cela passe alors par un important effort de formation et présuppose certaines qualités pédagogiques au sein du noyau fondateur:

On a été très très innovants dans ce domaine-là, puisqu'on a fait des formations, etc. Ce qui aujourd'hui commence à être un standard dans l'industrie, nous, on l'a imposé dès 1996, 1997. (fondateur)

$\boldsymbol{P 2}^{11}$ : À tous les stades, l'hypercroissance est favorisée par la priorité accordée au développement et à la valorisation de l'effort au sein du système de valeurs caractérisant le noyau fondateur du TMT.

Les fondateurs révèlent tous deux dans leur propos, à tous les stades du processus, une priorité accordée au développement et à la valorisation de l'effort au sein du système de valeurs. Efforts et développement de la croissance structurent ainsi leurs choix stratégiques, notamment en matière de financement:

C'est-à-dire que nous, on a deux choix. Soit on s'achète des Ferrari, soit on s'achète de la croissance, déjà. Donc on a toujours été assez paysans, là-dessus, avec [Pascal]. Et on a toujours financé notre propre croissance. On n'a jamais eu recours à l'emprunt, de façon significative. (fondateur)

La proposition P2 est donc corroborée. Plus précisément, le déclenchement et le maintien de l'hypercroissance, donc le développement de l'entreprise, sont les fruits (ou le prétexte) d'une combinaison d'un goût prononcé des fondateurs pour l'effort et d'autres valeurs qui les animent, telles que la passion et la curiosité. Le goût de l'effort contribue ainsi à entretenir le processus d'hypercroissance, parce qu'il est pour eux à l'origine d'un apprentissage, d'une acquisition de savoirs par «eux-mêmes»:

Je ne sais pas ce que c'est la trajectoire hypercroissance machin, mais on est partis de rien et on en est où on en est. Ça prend un certain temps et puis il faut l'apprendre et on l'apprend par soi-même, si on le fait faire par les autres, on n'apprend rien. (fondateur)

La passion et la curiosité animent également les fondateurs. Dans les propos des fondateurs coexistent ainsi systématiquement les notions de passion, d'amusement ("Ça nous amuse beaucoup avec [Pascal]. On est passionnés», [fondateur]) mais aussi de curiosité ("donc je suis curieux de voir où ça va nous mener parce que je pense que ça va nous mener plus grand, j'espère» [fondateur]).

11. La proposition P2 a été testée en croisant les codes VALORISATION DE SOI, EFFORT et les codes de STADES. 
La passion et la curiosité combinées au goût de l'effort vont permettre d'entretenir la croissance, car ils vont conduire les fondateurs à prendre des risques, à inventer et créer, même lorsque cela peut être considéré comme non raisonnable:

Une entreprise qui est inventive, qui crée, etc. Vous montrez le résultat à un financier, il va vous dire: "Mais qu'est-ce que c'est que cette entreprise? Les endroits où on investit, il faut arrêter les investissements parce que comme ça, ça va faire des dividendes supplémentaires.» Je m'en fous, nous on crée, donc il faut bien investir. (fondateur)

C'est, en outre, dans cette combinaison entre efforts exigés par de gros projets, développement important de l'entreprise et passion, que se créent et s'expriment le mieux les savoirs des fondateurs:

Il y a des métiers où on fera des plus gros projets probablement, on fera de la maîtrise d'offres sur des gros trucs-j'aimerais bien - parce que ça c'est passionnant. Parce que quand vous n'êtes pas maître d'œuvre, le projet n'est pas vraiment à vous, vous faites ce que le client vous dit de faire tandis que quand vous êtes maître d'œuvre, vous avez vraiment votre savoir, vous pouvez exprimer tout votre savoir. (fondateur).

Le déclenchement et le maintien de l'hypercroissance semblent ici, par conséquent, fondés sur la présence simultanée de mécanismes rationnels et cognitifs (l'effort à fournir pour le développement, l'accumulation de savoirs, l'exploitation des savoirs, les capacités) et de mécanismes plus irrationnels, tels que la passion, la curiosité, le plaisir et la valorisation de soi à travers l'expression de son savoir.

Je dirais qu'il faut une dose un peu de folie pour le faire, c'est-à-dire que je nous voyais bien tous les deux consultants avec [Julien] mais je n'imaginais pas spécialement embaucher, si l'on peut dire, et les choses se sont faites assez progressivement. Il y a une forme de surprise et de découverte, c'est-à-dire qu'il $y$ a toujours des gens qui font des business plans à l'infini et qui imaginent qu'ils vont dominer le monde, etc. Là, c'est plutôt une approche inverse, c'est-à-dire que c'est quand même une approche un peu folle, un peu passionnelle, avec une idée au départ et aussi des capacités. (cofondateur)

Les deux fondateurs de l'entreprise étudiée, qui concilient objectifs ambitieux de développement (ils veulent aller vers «Plus grand, plus gros, plus loin, plus de marchés», cofondateur) et les valeurs d'effort, de passion, de plaisir, sont donc bien des constructeurs de goodwill (au sens de Penrose, 1959). 


\subsubsection{Hypercroissance et échelons supérieurs: un processus marqué par l'inversion des causalités}

$P 3^{12}$ : L'expérience acquise dans l'avancement du processus de croissance conduit le noyau fondateur du TMT à percevoir la nécessité d'acquérir des ressources cognitives supplémentaires. Cela induit un changement de la composition, des compétences et de la répartition des rôles de l'équipe dirigeante.

Conformément à la proposition $\mathrm{P} 3$, nous observons une inversion du sens de causalité entre les caractéristiques du TMT et l'hypercroissance. L'expérience de l'avancement du processus de croissance ainsi que la volonté de son maintien conduisent le noyau fondateur à percevoir l'inadéquation du TMT sous sa forme initiale et la nécessité de le faire évoluer. Au moment du déclenchement, les caractéristiques cognitives du TMT sont une variable explicative centrale du processus de croissance. Ce dernier rétroagit au cours de son avancement pour influencer, à son tour, les caractéristiques des échelons supérieurs, retournant de la sorte l'approche initiale de l'UET, comme le suggère Hambrick (2007).

La première modification significative du TMT intervient à la suite des bouleversements de la structure du marché, devenu concurrentiel au tournant du millénaire. Le fondateur perçoit alors la nécessité de changer d'approche commerciale s'il veut continuer à croître, sans que les échelons supérieurs disposent à ce moment-là des compétences nécessaires en interne. Il est alors décidé d'élargir le TMT, afin d'y intégrer la ressource qui fait défaut:

Comme je ne savais pas faire, j'ai embauché mon frère. [...] je voyais que si notre marché français était saturé, pas saturé, mais... puisqu'on était mono-secteur, forcément, la croissance en France n'allait pas être gigantesque, puisque c'est un petit secteur. Donc, si on voulait croître, et rester mono-secteur, dans l'industrie de la santé, il fallait attaquer différents marchés à l'international. Donc, je voulais une expérience internationale, et une expérience de gens qui savaient traiter des gros projets et qui savaient avoir une approche commerciale intelligente, au-delà de la technique. On a réfléchi là-dessus. (fondateur)

L'arrivée du frère du fondateur correspond, donc, à un élargissement de la taille et des compétences du TMT. Élargissement et diversification de l'équipe dirigeante se doublent, par ailleurs, d'une réallocation des tâches parmi ses membres, de façon à permettre à chacun d'exploiter au mieux ses compétences distinctives. Ainsi, le cofondateur, qui est qualifié de technicien «de génie », avait cumulé, au fil du temps, un certain nombre de responsabilités commerciales, au point d'être détourné de son savoir-faire le plus précieux.

12. Analyse du texte correspondant aux codes EXPÉRIENCE CROISSANCE et CHANGEMENT ÉQUIPE, et cela, pour le stade AVANCEMENT. 
[Le cofondateur] s'occupait du commerce. C'était logique, puisqu'on était orienté technique. Donc, on vendait de la technique. Donc, le meilleur technicien... J'ai dit: «C'est pas possible, ça.» Je lui ai dit: «Si notre technique est la même que tout le monde, aujourd'hui, si on veut se différencier, il faut que [Pascal] revienne à la technique.» Donc, on a inventé un concept de recherche et de développement support, où, justement, comme on voulait taper du gros projet, il fallait qu'on soit capable de réaliser ces gros projets derrière. Il fallait que [Pascal] revienne dans l'ordre de la technique. (fondateur)

Notons que l'élargissement du TMT combiné à la réallocation des tâches en son sein, fruit de l'expérience de la croissance, opère ensuite comme un levier significatif du maintien d'un taux de croissance élevé : «ce qui devait se passer se passe finalement. [Arthur] trouve de gros projets, et [Pascal] les réalise» (fondateur).

L'intégration d'une compétence commerciale au sein du TMT n'est cependant pas la seule modification intervenue avec l'avancement du processus de croissance. À partir d'une certaine taille, le besoin d'étoffer la direction d'une forte compétence financière se fait sentir.

[L'entreprise] passait un palier en matière de taille et avait besoin de procédures plus formalisées. Elle passait de la petite entreprise à l'entreprise moyenne, c'est-à-dire le passage des 150 salariés. Nous avions une équipe qui savait gérer l'entreprise entre 50 et 150 salariés mais qui ne pouvait plus le faire au-delà. (DAF)

La nécessité du recrutement d'un directeur financier de haut niveau pour élargir le TMT a été apprise dans la douleur, après avoir fait l'expérience de plusieurs échecs. Conformément à la proposition P3, on peut donc affirmer que cette nécessité perçue et les transformations conséquentes de l'équipe dirigeante résultent de l'expérience acquise dans l'avancement du processus d'hypercroissance.

Je ne sais pas si on aurait la même robustesse, par exemple au niveau fiscal ou au niveau social, si on ne nous avait pas plantés plusieurs fois. Parce que maintenant, on a su mettre en place et trouver des vrais professionnels. (cofondateur)

$P^{13}$ : L'hypercroissance induit un haut degré d'exigence pour l'accomplissement des tâches administratives (task challenge), faisant ressentir un besoin de recrutement pour l'équipe managériale. Le haut degré d'exigence est source

13. Le test de la proposition $\mathrm{P} 4$ consiste à analyser la présence du code EXIGENCE DE LA FONCTION MANAGÉRIALE IMPOSÉE PAR LA CROISSANCE dans chacune des étapes du processus d'hypercroissance (déclenchement et avancement) ainsi que son lien avec le code PERCEPTION DE LA NÉCESSITÉ de RECRUTEMENT. 
d'erreurs dans la prise de décision car, par manque de temps pour consolider leur expérience, les membres du TMT en place opèrent des "raccourcis mentaux » dans leur prise de décision (Hambrick et al., 2005).

L'analyse des données nous permet de constater que c'est dans la phase d'avancement du processus d'hypercroissance que l'exigence de la fonction managériale liée à la croissance est la plus forte (8 occurrences sur 8$)$, même si, dans le cas de l'entreprise étudiée, il semble que ce soit dès le déclenchement de l'hypercroissance que les membres du noyau fondateur aient eu conscience de cette exigence.

L'exigence pour l'accomplissement des tâches administratives est perçue comme particulièrement pressante. Les cofondateurs évoquent que «très vite» ils ont essayé d'y répondre. Ils parlent d'une «croissance usante», «stressante», d' «années douloureuses». Le coût psychologique est donc non négligeable.

L'exigence liée aux tâches administratives est plus particulièrement ressentie par le noyau fondateur lorsque l'organisation connaît des seuils organisationnels :

On a fait des erreurs, on a mis en place un modèle et, en fait, il a fallu le casser à chaque fois qu'on a passé des étapes. Il y a eu un premier modèle qui a dîu exister jusqu'à 10, après il y a eu un deuxième modèle à 20, après on peut dire vers 40-50, après à 100, après à 150, 200. (cofondateur)

Les tâches administratives imposées par l'hypercroissance sont complexes. Cette complexité est due à la fois à la nature des tâches à accomplir et à leur imbrication, au caractère évolutif des exigences de la fonction managériale au fur et à mesure que la taille s'accroît (et elle s'accroît rapidement) et au degré d'intégration sociale requis pour accomplir ces tâches efficacement.

Des tâches administratives transversales ainsi que des tâches administratives plus fonctionnelles sont évoquées par l'équipe dirigeante. Parmi les tâches administratives transversales qui incombent à l'équipe dirigeante sont nommées notamment la tâche de «mixer» des compétences diverses, telles que des compétences technologiques maîtrisées par l'organisation avec des compétences métier, plus longues à acquérir; celle de «procéder à une spécialisation des membres de l'équipe» et celle de «déléguer le management». Elles incombent avant tout aux fondateurs. Des tâches plus fonctionnelles sont également évoquées : embaucher, gérer la paie, gérer la trésorerie, contrôler. Ces tâches administratives fonctionnelles s'enchaînent et interagissent: 
Le deuxième point après, c'est qu'à partir du moment où on embauche, derrière, il faut facturer et après il faut être capable de gérer la trésorerie. Il y a cette partie-là qui est vraiment sensible et on a cru que ça marchait bien et un beau jour, on a découvert que ça marchait très, très mal.

L'accomplissement des tâches multiples, imbriquées et évolutives, qui incombe au TMT, suppose l'existence d'une équipe «solide», possédant des aptitudes particulières, ce qui accroît d'autant la perception d'un degré élevé d'exigence de la fonction managériale:

Il faut avoir une équipe solide avec un niveau suffisant pour pouvoir continuer dans le temps, mais le malheur, c'est que votre comptable ne peut pas être votre gestionnaire financier, et votre gestionnaire financier ne peut pas être votre directeur financier, sauf si vous avez eu la chance de tomber sur la personne exceptionnelle qui est capable. (fondateur)

Il y a donc une perception des dirigeants-fondateurs d'une nécessaire évolution des contours du TMT et des rôles accomplis en son sein:

Nous étions tous les deux, puis après on a dit: «Oui, il faut quelqu'un qui nous aide pour s'occuper de la facturation etc.» parce qu'avant on était consultants. On était consultants parce qu'il y avait d'autres personnes qui s'occupaient de la facturation, puis il a fallu faire un peu de marketing et c'est comme ça qu'une première personne est venue nous «supporter» d'un point de vue administratif, puis on a commencé à embaucher un consultant, puis un deuxième. (cofondateur)

La proposition 4 selon laquelle l'hypercroissance induit un haut degré d'exigence pour l'accomplissement des tâches administratives, exigence qui façonne la perception d'une nécessaire évolution des contours du TMT et des rôles accomplis en son sein, est par conséquent corroborée. La perception des besoins fonctionnels et des profils adaptés s'est cependant forgée «dans la douleur». En effet, par manque d'une expérience «héritée» dans ce domaine, un certain nombre d'erreurs aux conséquences lourdes à assumer ont été commises lors du recrutement de managers clés. Tel a notamment été le cas de la fonction finance, avant de trouver finalement le profil adéquat. Il en résulte que, dans une période d'hypercroissance, l'apprentissage de l'équipe dirigeante s'avère particulièrement coûteux sur le plan psychologique. Le fait d'être constamment sous pression à cause de la croissance rapide et de devoir en même temps assumer les conséquences de certaines erreurs, de recrutement de nouveaux managers entre autres, est une source potentielle de pénibilité. 


\section{Discussion et conclusion}

Cette étude montre, en premier lieu, qu'il existe une interaction étroite entre hypercroissance et dynamique de l'équipe de direction, et ce, à tous les stades de l'hypercroissance.

Nous observons effectivement, comme nous y invite la théorie des échelons supérieurs, l'importance des expériences détenues par le noyau fondateur au moment de la création de l'entreprise, ainsi que des visions et valeurs de ses membres, pour déclencher un mouvement d'hypercroissance. L'expérience vécue (et donc acquise) dans l'avancement de cette forte croissance influence cependant, à son tour, la cognition du TMT et conduit à une modification des caractéristiques de ce dernier. On constate notamment une augmentation significative du degré d'hétérogénéité fonctionnelle de ses membres au cours du temps, comme conséquence de la nécessité perçue d'une professionnalisation croissante des services managériaux à accomplir. Au-delà de la diversification des expériences fonctionnelles liée au recrutement de nouveaux membres du TMT (expériences héritées de l'extérieur, au sens de Penrose), nous enregistrons également une dynamique de réallocation des tâches parmi les membres en place en fonction de l'expérience acquise au cours du processus de croissance, ce qui démontre la pertinence d'un recours aux explications de Penrose (1959). Nous avons ainsi pu démontrer une inversion de la causalité entre les caractéristiques du TMT et la dynamique de la croissance, confirmant l'intuition de l'intérêt d'une inversion du schéma explicatif initial de l'UET, telle qu'elle a été exprimée par Hambrick (2007). Cela montre, en outre, la pertinence de l'adoption d'une approche processuelle par étude de cas pour analyser les liens entre les caractéristiques cognitives et les valeurs de l'équipe dirigeante, d'une part, et les résultats organisationnels en termes de croissance, d'autre part. L'étude de cas nous permet de dépasser le simple constat d'une cooccurrence entre un niveau d'hétérogénéité donné et la croissance fait dans l'UET, pour étudier le processus même de diversification des profils fonctionnels (et des caractéristiques cognitives, plus globalement) comme le résultat d'exigences de la fonction managériale particulièrement élevées en cas d'hypercroissance. Autrement dit, contrairement à la plupart des études empiriques liées à la perspective UET, nous n'étudions pas la corrélation, en coupe instantanée, entre divers degrés d'hétérogénéité au niveau des variables démographiques et cognitives et le taux de croissance, mais le processus d'augmentation d'une telle hétérogénéité. Cette approche permet alors de mettre en évidence un schéma séquentiel de diversification des expériences fonctionnelles et de la professionnalisation des services managériaux correspondants. L'élargissement du TMT commence ainsi avec le recrutement d'un directeur commercial 
(expérience fonctionnelle output) et se poursuit, dans un deuxième temps, avec l'intégration d'un directeur financier (expérience fonctionnelle périphérique). La question du recrutement d'un DRH est posée, ensuite.

Cette étude suggère, en second lieu, que l'apprentissage collectif de l'équipe de direction est une condition sine qua non du maintien d'une hypercroissance soutenue et durable. Plus que l'hétérogénéité ou l'homogénéité de l'équipe (Hambrick et al., 1996), ce serait peut être la capacité d'apprentissage de l'équipe, façonnée par la croissance, qui pourrait expliquer la croissance de la firme.

Elle montre, enfin, que cet apprentissage reste coûteux psychologiquement et financièrement, notamment pour les membres fondateurs de l'équipe de direction. Cela pourrait expliquer les difficultés de nombre de PME à gérer leur processus d'hypercroissance, ou encore la réticence de certaines entrepreneurs à faire croître leur entreprise.

\section{Bibliographie}

AmASON, A.C. (1996), «Distinguishing the effects of functional and dysfunctional conflict on strategic decision making: resolving paradox for top management teams", Academy of Management Journal, vol. 39, n¹, p. 123-148.

Betbèze, J.-P. et C. SAint-ÉTIENNE (2006), Une stratégie PME pour la France: favoriser l'essor des gazelles, entreprises moyennes en forte croissance, Rapport du CAE, n ${ }^{\circ} 61$, Paris, La Documentation française, 165 p.

BOEKER, W. (1997), «Strategic change: the influence of managerial characteristics and organizational growth», Academy of Management Journal, vol. 40, n 1 , p. $152-170$.

BOURGEOIS, L. et K. EISENHARDT (1988), «Strategic processes in high velocity environments: four cases in the microcomputer industry», Management Science, vol. $34, \mathrm{n}^{\circ}$ 7, p. 816-835.

CARPenter, M.A., M.A. GeletKanYCZ et W.K.G. SANDERS (2004), «Upper echelons research revisited: antecedents, elements, and consequences of top management team composition », Journal of Management, vol. 30, $\mathrm{n}^{\circ} 6$, p. $749-778$.

EISENHARDT, K.M., C.B. SCHOONHOVEN et C. BIRD (1990), «Organizational growth: linking founding team, strategy, environment and growth among U.S semiconductor ventures, 1978-1988», Administrative Science Quarterly, vol. 35, $\mathrm{n}^{\circ} 3$, p. $504-529$.

ENGEL, D. (2002), «The impact of venture capital on firm growth: an empirical investigation », ZEW Discussion paper $\mathrm{n}^{\circ} 02-02,30 \mathrm{p}$. 
ENGEL, D. et M. KeILBACH (2007), «Firm-level implications of early-stage venture capital investment: an empirical investigation», Journal of Empirical Finance, vol. 14 , p. $150-167$.

FinKELSTEIN, S. et D.C. HAMBRICK (1990), «Top-management team tenure and organizational outcomes: the moderating role of managerial discretion », Administrative Science Quarterly, vol. 35, $\mathrm{n}^{\circ} 3$, p. 484-503.

Glachant, J., J.-H. Lorenzi et P. Trainar (2008), Private Equity et capitalisme français, Rapport du CAE, Paris, La Documentation française, $329 \mathrm{p}$.

GREVE, H.R. et H. MitsuHASHI (2008), «Power and glory: concentrated power in top management teams », Organization Studies, vol. 28, n 8, p. 1197-1221.

HAMBRICK, D.C. (2007), «Upper echelons theory: an update», Academy of Management Review, vol. 32, n² 2 , p. 334-343.

HAMBRICK, D.C., T.S. CHO et M.-J. CHEN (1996), «The influence of top management team heterogeneity on firms' competitive moves », Administrative Science Quarterly, vol. 41, n 4, p. 659-684.

HAMBRICK, D.C. et L.M. CROZIER (1985), «Stumblers and stars in the management of rapid growth », Journal of Business Venturing, vol. 1, n 1 , p. 31-45.

HAMBRICK, D.C., D. FinKElSTEIN et A.C. MOONEY (2005), «Executive jobs demands: new insights for explaining strategic decisions and leader behaviours », Academy of Management Review, vol. 30, n³ 3, p. 472-493.

HAmbrick, D.C., M.A. GeletKanyCZ et J.W. Fredrickson (1993), «Top executive commitment to the status quo: some tests of its determinants », Strategic Management Journal, vol. 14, n 3, p. 401-418.

HAMBRICK, D.C. et P.A. MASON (1984), «Upper echelons: the organization as a reflection of its top managers », Academy of Management Review, vol. 9, n², p. 193-206.

Kilduff, M., R. ANGLEMAR et A. MeEHRA (2000), «Top management-team diversity and firm performance : examining the role of cognitions », Organization Science, vol. 11, no 1, p. 21-34.

KOR, Y.Y. (2003), «Experience-based top management team competence and sustained growth », Organization Science, vol. 14, nº 6, p. 707-719.

LIN, H.-C. et C.-T. SHIH (2008), «How executive SHRM system links to firm performance: the perspectives of upper echelon and competitive dynamics », Journal of Management, vol. 3, $\mathrm{n}^{\circ}$ 5, p. 853-881.

Miles, M. et A.M. Huberman (1994), Qualitative Data Analysis, 2e édition, Thousand Oaks, Sage, 338 p.

Olson, B.J, S. PARAYITAM et Y. BAO (2007), «Strategic-decision-making: the effects of cognitive diversity, conflict and trust on decision outcomes », Journal of Management, vol. 33, $\mathrm{n}^{\circ}$ 2, p. 196-222.

Penrose, E.T. (1959), The Theory of the Growth of the Firm, Oxford, Basil Blackwell, $272 \mathrm{p}$. 
Peterson, R.S., P.V. Martorana, D.B. Smith et P.D. Owens (2003), «The impact of chief executive officer personality on top management team dynamics: one mechanism by which leadership affects organizational performance», Journal of Applied Psychology, vol. 88, n 5, p. 795-819.

SAPIENZA, H. et S. MANIGART (1996), «Venture capitalist governance and value added in four countries », Journal of Business Venturing, vol.11, n ${ }^{\circ}$, p. 439-469.

Smith, K.G., K.A. Smith, J.D. Olian, H.P. Sims, D.P. O’BAnNON et J.A. SCUlly (1994), «Top management team demography and process: the role of social integration and communication», Administrative Science Quarterly, vol. 39, $\mathrm{n}^{\circ} 3$, p. 412-438.

WEINZIMMER, L.G. (1997), «Top management team correlates of organizational growth in a small business context: a comparative study », Journal of Small Business Management, vol. 35, $\mathrm{n}^{\circ} 3$, p. 1-10.

WirTz, P. (2009), «Do high-growth entrepreneurial firms have a specific system of governance?», FARGO working paper series, $41 \mathrm{p}$. 